\title{
The benefits and harms of Chinese medicinal herbs for the treatment of rapidly progressive glomerulonephritis in adult patients
}

\author{
Jialing LIU ${ }^{1,2 *} \mathbb{\infty}$, Xiaoxi ZENG ${ }^{3,4 *}$, Qizhan LIN $^{5,6}$, Weifeng HE7 , Wei YE ${ }^{8}$, Qianchun YANG ${ }^{9,10 *}$
}

\begin{abstract}
Objective: The aim of this review was to assess the benefits and harms of Chinese medicinal herbs for the treatment of rapidly progressive glomerulonephritis (RPGN) in adult patients. Methods: Our study included only randomized controlled trials (RCTs). We designed a strategy for searching the EMBASE, CENTRAL, PubMed, CBM, CNKI, and VIP. All studies were analyzed using the criteria of the Cochrane Handbook. All studies included were assessed the risk of bias. Review Manager ver. 5.3.5 was used for the data analysis. GRADE profiler was used to evaluate quality. Results: Two studies were studied (74 Chinese participants were included). We found that compared with supportive therapies combined with specific therapies, Chinese medicinal formulae plus supportive therapies combined with specific therapies significantly reduced the serum creatinine levels (SCr; two studies, 62 participants: mean difference (MD), $-145.93 \mu \mathrm{mol} / \mathrm{L} ; 95 \%$ confidence interval (CI), -236.75 to $\left.-55.11 ; \mathrm{I}^{2}=0 \%\right)$ and utilization number of methylprednisolone and prednisone (two studies, 74 participants: $\mathrm{MD}$, $-1.64 \mathrm{~g}$; $95 \% \mathrm{CI},-1.87$ to -1.40 ; $\left.\mathrm{I}^{2}=0 \%\right)$. Conclusion: Our study suggested that there were insufficient evidences to confirm that the use of Chinese medicinal formulae for adults with RPGN was beneficial and safe.
\end{abstract}

Keywords: chinese medicinal herbs; rapidly progressive glomerulonephritis; supportive therapy; specific therapy.

Practical Application: Our study suggested that there were insufficient evidences to confirm that the use of Chinese medicinal formulae for adults with rapidly progressive glomerulonephritis (RPGN) was beneficial and safe. Larger, multicenter studies of high methodological quality are needed to further examine the usefulness of Chinese medicinal herbs for the treatment of RPGN in adults.

\section{Introduction}

Rapidly progressive glomerulonephritis (RPGN), also known as crescentic glomerulonephritis, is characterized by the presence of extensive glomerular crescents (normally affecting more than $50 \%$ of the glomeruli) as the main histological finding and rapid decline in kidney function, which may complicate glomerular disease (Couser, 1988; Jennette, 2003; Nachman et al., 2011; Succar et al., 2016; Parmar \& Bhimji, 2018). RPGN can be classified into secondary and primary types. Secondary RPGN may be associated with systemic necrotizing vasculitis, systemic lupus erythematosus (SLE), relapsing polychondritis, mixed immunoglobulin $\mathrm{G}$ and immunoglobulin $\mathrm{M}$ (IgG-IgM) cryoglobulinemia, rheumatoid vasculitis, malignancy, and the use of drugs such as penicillamine, hydralazine, allopurinol (with vasculitis), rifampin, antithyroid agents, and aminoguanidine (Parmar \& Bhimji, 2018). Primary RPGN can be related to infective endocarditis, post-streptococcal glomerulonephritis, hepatitis B infection, occult visceral sepsis, and another primary glomerular disease such as membranous glomerulonephritis, membranoproliferative glomerulonephritis type II, or immunoglobulin A ( $\operatorname{Ig} \mathrm{A})$ nephropathy.

Idiopathic or primary RPGN includes five types (Couser, 1988; Jennette, 2003; Nachman et al., 2011; Parmar \& Bhimji, 2018; Moroni \& Ponticelli, 2014): type I presents linear deposits of IgG; type II presents granular deposits of immunoglobulin; type III presents few or no immune deposits, pauci-immune antineutrophil cytoplasmic antibody (ANCA)-related minute vessel vasculitis, which may be limited to the kidney or part of a systemic disease including granulomatosis with polyangiitis or microscopic polyangiitis; type IV is a combination of types I and III; and type V is ANCA-negative pauci-immune kidney vasculitis (5-10\% of all cases). The clinical signs and symptoms of idiopathic

${ }^{1}$ The Second Clinical Medical College, Guangzhou University of Chinese Medicine, Guangzhou, China ${ }^{2}$ Department of Nephrology, Guangdong Provincial Hospital of Chinese Medicine, Guangzhou, China

${ }^{3}$ Kidney Research Institute, Department of Nephrology, West China Hospital, Sichuan University, Chengdu, China

${ }^{4}$ West China Biomedical Big Data Center, West China Hospital, Sichuan University, Chengdu, China

${ }^{5}$ Department of Hemodialysis, Guangdong Provincial Hospital of Chinese Medicine, Guangzhou, China

${ }^{6}$ Department of Hemodialysis, The Second Affiliated Hospital, Guangzhou University of Chinese Medicine, Guangzhou, China

Department of Oncology, The Second People's Hospital of Hunan Province, Changsha, China

${ }^{8}$ Department of Neonatology, Renmin Hospital of Wuhan University, Wuhan, China

${ }^{9}$ Guangzhou University of Chinese Medicine, Guangzhou, China

${ }^{10}$ Second Outpatient Department, First Affiliated Hospital, Guangzhou University of Chinese Medicine, Guangzhou, China

\#Co-first author, these authors contributed equally to this work.

*Corresponding author: qcyang@yandex.com 
RPGN include weakness, anemia, nausea, hematuria, vomiting, oliguria, fluid retention, and uremia (Parmar \& Bhimji, 2018). Idiopathic RPGN accounts for less than $10 \%$ of cases of primary glomerulopathy. However, more than $50 \%$ of patients with RPGN develop acute nephritic syndrome and kidney function deteriorates rapidly. RPGN is relatively rare and is observed in $2-13 \%$ of kidney biopsies (Schena, 1997; Liu et al., 2004; Quiroga et al., 2015; Okpechi et al., 2016; Perkowska-Ptasin ska et al., 2017). However, RPGN patients have a high risk of developing end-stage kidney disease (ESKD) (Chembo et al., 2015; van Daalen et al., 2018). Patients with anti-glomerular basement membrane (anti-GBM) disease plus anti-related RPGN have an extremely poor prognosis (Arimura et al., 2016). A study conducted in New Zealand found that more than $60 \%$ of patients with RPGN had ESKD at the 10-year follow up (Chembo et al., 2015).

Treatment for RPGN includes supportive and specific therapies. Supportive therapies include infection control, control of volume status (i.e., providing dialysis if required), and smoking cessation (Parmar \& Bhimji, 2018). Specific therapies involve the induction and maintenance of remission. The initial treatment typically involves administration of glucocorticoids and cyclophosphamide, which induces remission in 85-90\% of patients within 2 to 6 months and complete remission in about $75 \%$. For patients with rapidly progressive crescentic IgA nephritis, anti-GBM glomerulonephritis, Henoch-Schönlein purpura nephritis, segmental necrotizing glomerulonephritis, and pauci-immune focal glomerulonephritis, the initial treatment is a combination of cytotoxic drugs and corticosteroids (Parmar \& Bhimji, 2018; Cattran et al., 2012). Moreover, plasma exchange may be useful for patients with advanced kidney failure (serum creatinine $[\mathrm{SCr}]>500 \mu \mathrm{mol} / \mathrm{L}$ and/or requiring dialysis) or severe pulmonary hemorrhage, which is related to anti-GBM disease (Parmar \& Bhimji, 2018; Cattran et al., 2012). However, relapse is common, particularly in patients with microscopic polyangiitis. The current treatment outcomes for RPGN are poor, and many patients ultimately require renal transplantation or long-term dialysis (Kannan \& Mattoo, 2001).

Several traditional Chinese medicines (TCMs) have immunosuppressive activity, and Chinese medicinal herbs as adjuvants to RPGN therapy have been proved to have beneficial effects (Deng et al., 2004; Wang et al., 2008). According to TCM theory, the RPGN is equivalent to hematuria (xue niao: the discharge of bloody urine or urine tinged with blood without pain during urination), edema (shui zhong: defined as any disease characterized by subcutaneous fluid retention), vomiting (ou tu: a disease status characterized by compulsive expulsion of gastric contents through the mouth), and dribbling urinary block disease (long bi: such as complete blockage of urine flow or dribbling urination). Nelumbo nucifera ( $N$. nucifera), an edible Chinese medicinal herb, is widely used to treat inflammation and hemostasis (Liu et al., 2006; Liu et al., 2007; Ka et al., 2010). A recent study found that (S)-armepavine, a vital bioactive compound in N. nucifera, slowed the progression of system lupus erythematosus (SLE) in $\mathrm{MRL} /$ lpr mice by reducing glomerular immune deposits and mesangial cell hypercellularity (Liu et al., 2007). Another study found that the most common TCM syndromes associated with RPGN included dampness-heat and blood stasis syndrome, kidney yin deficiency and blood stasis syndrome, and spleen and kidney deficiency syndrome (Chen, 2005). Based on pattern differentiation, several Chinese medicinal herbs combined with western medicine showed to have positive effects in patients with RPGN via activation of NF- $\kappa \mathrm{B}$, modulation of T/B cell activity, and protection of kidney and spleen cells (Deng et al., 2004; Wang et al., 2008; Ka et al., 2010; Xu, 1999; Zhong, 2002; Zhou \& Zhou, 2003; Wang, 2003; Wang \& Chen, 2004).

TCM, which has been widely used all over the world, has shown therapeutic efficacy for RPGN. However, the safety and efficacy of TCM have not been systematically studied. Therefore, the purpose of this review was to access the benefits and harms of Chinese medicinal herbs for the treatment of RPGN in adult patients.

\section{Methods}

\subsection{Criteria for considering studies for this review}

\subsubsection{Participants}

Only randomized controlled trials (RCTs) were included in our review. We included studies in which the patients (included men and women) who were aged more than 18 years old with a histologically confirmed diagnosis of RPGN (the presence of cellular or fibrocellular crescents in more than $50 \%$ of the glomeruli), and an diagnosis of RPGN defined by the authors. Patients who were pregnant or had sclerotic glomerulonephritis or secondary forms of RPGN (e.g., systemic necrotizing vasculitis, systemic lupus erythematosus, essential mixed IgG-IgM cryoglobulinemia, Henoch-Schönlein purpura, rheumatoid vasculitis, relapsing polychondritis, and malignancy were excluded.

\subsubsection{Interventions}

Our review included studies in which the authors compared the efficacy of Chinese medicinal herbs and placebo, no treatment, supportive therapies (control of infection and volume status, and smoking cessation), specific therapies (glucocorticoids combined with cytotoxic drugs and plasma exchange), or combined supportive and specific therapies; Chinese medicinal herbs combined with supportive or specific therapies and supportive or specific therapies alone; Chinese medicinal herbs plus supportive combined with specific therapies and combined supportive and specific therapies alone; and Chinese medicinal herbs plus combined supportive and specific therapies and placebo plus combined supportive and specific therapies.

\subsubsection{Observed indicators}

The primary observed indicators included all-cause mortality, ESRD or renal replacement therapy, and oliguria or anuria remission (defined in the individual studies). The secondary observed indicators included kidney function (indexed by SCr and blood urea nitrogen (BUN) levels), liver function (indexed by aspartate transaminase (AST) or alanine transaminase (ALT) levels), time for the 24-h urine volume to return to normal, anemia correction (indexed by hemoglobin $(\mathrm{Hb})$ and hematocrit 
(HCT) levels), number of immunosuppressive drugs used (including prednisone, cyclophosphamide, methylprednisolone, and mycophenolate mofetil), quality of life, TCM tongue and pulse assessment (tongue coating and pulse), economic index, adverse events, and rate of withdrawals from the study.

\subsubsection{Search methods for identification of studies}

The databases listed below using a series of related terms were searched. The searches ended on December 31, 2018. The databases included EMBASE (from 1967 to December 2018), PubMed (from 1966 to December 2018), the Cochrane Central Register of Controlled Trials (from 1966 to December 2017), Chinese National Knowledge Internet (CNKI) (from 1979 to December 2018), Chinese Biomedicine Database (CBM) (from 1978 to December 2018), and Chinese Science and Technology Periodical Database (VIP) (from 1989 to December 2018). All studies included were analyzed according to Cochrane Hand Book criteria. The following search terms were used: (Traditional Chinese Medicine OR Chinese Medicine OR TCM OR Traditional Medicine OR Chinese and Western Medicine OR Traditional Chinese Pharmacy OR Integrated Chinese and Western Medicine OR Chinese Patent Medicine OR Chinese Traditional Patent Medicine OR Chinese traditional herbs OR Chinese medicinal herbs OR Chinese herbal medicines) AND (rapidly progressing glomerulonephritis OR crescentic glomerulonephritis OR RPGN OR Rapidly progressive glomerulonephritis OR Rapidly Progressive GN OR acuterapidly progressive glomerulonephritis OR CREGN OR crescent nephritis OR necrotizing crescentic glomerulonephritis). We manually searched the Journal of Guangzhou University of Traditional Chinese Medicine. We attempted to contact all the original authors to obtain study protocols for review.

\subsection{Data collection and analysis}

\subsubsection{Selection of studies}

Two reviewers independently evaluated the titles and abstracts of studies obtained by performing the comprehensive literature search. Excluded studies were listed with reasons for exclusion. The studies that might be eligible for inclusion were initially retained, and full texts were retrieved and scanned. Original authors were contacted to obtain missing information. The two reviewers independently determined the included studies. Disagreements were resolved by arbitration of a third reviewer.

\subsubsection{Data extraction and management}

For each qualified literature, two reviewers independently extracted data using standard data-extraction forms. The following information was extracted: the first author's name, year of publication, country where the study was conducted, study design and settings, number of participants, demographic and clinical characteristics of participants, interventions, outcomes, and other information which might help evaluate bias and conflicts of interest. If data from the published articles were insufficient, the original authors were contacted for additional information. We extracted the contents of Chinese herbal formulation of the included studies, and the names of the herbs were provided in three languages (Chinese, Latin, and English) in Table 1.

\subsubsection{Assessment of risk of bias in included studies}

The following items were independently assessed by two authors (YQC and HWF) using the risk of bias evaluated tool (Higgins \& Green, 2011). Whether the allocation was concealed adequately (selection bias). Whether there was adequately sequence generation (selection bias). Whether partial outcome data were adequately addressed (attrition bias) (Participants and personnel and Outcome assessors). Whether knowledge of the allocated interventions was adequately prevented during the study (detection bias). Whether the study was distinctly free of other problems which could put it on a risk of bias. Whether reports of study were free of recommendation of selective outcome reporting (reporting bias).

\subsubsection{Measures of treatment effect}

The data were expressed as risk ratios (RRs) with 95\% confidence interval (CI) for dichotomous variables (RPGN remission, mortality, adverse effects, and ESKD) and mean differences (MD) with 95\% CI for continuous variables (SCr, BUN, $\mathrm{Hb}, \mathrm{HCT}$, and time for 24-h urine volume to return to normal).

\subsubsection{Dealing with missing data}

We contacted original authors by phone to obtain missing data. We received the study information for the included studies. We investigated attrition rates, including losses to follow-up, withdrawals, and dropouts. Issues elevated by missing data and the imputation means used were critically evaluated (Liu et al., 2006).

\subsubsection{Assessment of heterogeneity and reporting biases}

The chi-square test was used to detect heterogeneity among studies. $p$-values $<0.05$ was considered as statistical significance. Additionally, the $\mathrm{I}^{2}$ index was used to quantify the degree of heterogeneity. $\mathrm{I}^{2}$ values of $0-25 \%, 50 \%$, and $75 \%$ were defined as low, moderate, and high heterogeneity, respectively (Higgins \& Thompson, 2002). In particular, funnel plots had been initially planned to assess the reporting bias, but they were not performed because there was only two studies included.

\subsubsection{Data synthesis}

Data were merged using a random-effects model under the hypothesis that effects may differ across studies.

\subsubsection{Subgroup analysis and investigation of heterogeneity}

The following subgroup analyses were planned to evaluate the effects of Chinese medicinal herbs under different conditions and to explore the sources of heterogeneity: different pathological categories (i.e., Types I to V RPGN), different underlying kidney diseases (i.e., systemic vasculitis, anti-GBM disease, lupus nephritis, IgA nephropathy, etc.), different $\mathrm{CMH}$ prescriptions, and different durations of therapy and follow up. 
Table 1. Contents of the formulations (in three languages) used in the included studies.

\begin{tabular}{|c|c|c|}
\hline Study ID & Herbs (Composition) in three language & $\begin{array}{c}\text { Methods of } \\
\text { administration }\end{array}$ \\
\hline $\begin{array}{l}\text { Deng et al. } \\
\text { (2004) }\end{array}$ & $\begin{array}{l}\text { Self-Developed clear hear and activate blood prescription: Baihuasheshecao (Herba Hedyotidis Diffusae / Spreading } \\
\text { Hedyotis Herb), Rendongteng (Caulis Lonicerae / Japanese Honeysuckle Stem), Zihuadiding (Herba Violae / } \\
\text { Philippine Violet Herb), Chishao (Radix Paeoniae Rubra / Red Paeony Root), Shengdi (Radix Rehmanniae / } \\
\text { Rehmannia Root), Huangjing (Rhizoma Polygonati / Manyflower Solomonseal Rhizome / Siberian Solomonseal } \\
\text { Rhizome / King Solomonseal Rhizome), Dangshen (Radix Codonopsis / Pilose Asiabell Root / Moderate Asiabell } \\
\text { Root / Szechwon Tangshen Root), Danshen (Radix Salviae Miltiorrhizae / Danshen Root), Zhidahuang (Radix } \\
\text { Rumicis / Dock Root), Huoxiang (Herba Agastaches / Wrinkled Gianthyssop Herb). }\end{array}$ & $\begin{array}{c}\text { Oral } \\
\text { administration }\end{array}$ \\
\hline $\begin{array}{l}\text { Wang et al. } \\
\text { (2008) }\end{array}$ & $\begin{array}{l}\text { 1.Pattern of accumulation of dampness-heat and blood stasis in the kidney collateral: Baihuasheshecao (Herba } \\
\text { Hedyotidis Diffusae / Spreading Hedyotis Herb), Pugongying (Herba Taraxacig / Mongolian Dandelion Herb), } \\
\text { Banzhilian (Herba Scutellariae Barbatae / Barbed Skullcap Herb), Rendongteng (Caulis Lonicerae / Japanese } \\
\text { Honeysuckle Stem), Zihuadiding (Herba Violae / Philippine Violet Herb), Danshen (Radix Salviae Miltiorrhizae } \\
\text { / Danshen Root), Chishao (Radix Paeoniae Rubra / Red Paeony Root), Taoren (Semen Persicae / Peach Seed), } \\
\text { Shengdi (Radix Rehmanniae / Rehmannia Root), Mugua (Fructus Chaenomelis / Common Floweringquince } \\
\text { Fruit), Zhidahuang (Radix Rumicis / Dock Root), Gancao (Radix Glycyrrhizae / Liquoric Root); 2. Pattern of } \\
\text { kidney yin deficiency and blood stasis in the kidney collateral: Shengdi (Radix Rehmanniae / Rehmannia Root), } \\
\text { Maidong (Radix Ophiopogonis / Dwarf Lilyturf Tuber), Shanzhuyu (Fructus Corni / Common Macrocarpium } \\
\text { Fruit), Huangjing (Rhizoma Polygonati / Manyflower Solomonseal Rhizome / Siberian Solomonseal Rhizome / } \\
\text { King Solomonseal Rhizome), Shihu (Herba Dendrobii / Dendrobium), Guiban (Carapax et Plastrum Testudinis } \\
\text { / Tortoise Shell), Zhimu (Rhizoma Anemarrhenae / Common Anemarrhena Rhizome), Duzhong (Cortex } \\
\text { Eucommiae / Eucommia Bark), Huangbai (Cortex Phellodendri / Amur Corktree Bark), Danshen (Radix Salviae } \\
\text { Miltiorrhizae / Danshen Root), Chishao (Radix Paeoniae Rubra / Red Paeony Root), Zhidahuang (Radix Rumicis } \\
\text { / Dock Root); 3.Pattern of dual deficiency of the spleen-kidney and blood stasis in the kidney collateral: Huangqi } \\
\text { (Radix Astragali / Root), Huaishanyao (Rhizoma Diosscoreae / Common Yam Rhizome / Wingde Yan Rhizome), } \\
\text { Chuanxiong (Rhizoma Chuanxiong / Szechuan Lovage Rhizome), Gegen (Radix Puerariae / Kudzuvine Root), } \\
\text { Huangjing (Rhizoma Polygonati / Manyflower Solomonseal Rhizome / Siberian Solomonseal Rhizome/King } \\
\text { Solomonseal Rhizome), Shanzhuyu (Fructus Corni / Common Macrocarpium Fruit), Xianlingpi (Herba Epimedii / } \\
\text { Epimedium Herb), Bajitian (Radix Morindae Officinalis / Medicinal Indianmulberry Root), Taoren (Semen Persicae } \\
\text { / Peach Seed), Honghua (Flos Carthami / Safflower), Baizhu (Rhizoma Atractylodis Macrocephalae / Largehead } \\
\text { Atractylodes Rhizome), Zhidahuang (Radix Rumicis / Dock Root). }\end{array}$ & $\begin{array}{c}\text { Oral } \\
\text { administration }\end{array}$ \\
\hline
\end{tabular}

\subsubsection{Sensitivity analysis}

We performed a sensitivity analysis that involved removing studies with a high risk of bias and repeating the meta-analysis. A fixed-effects model was used to determine the robustness of the results.

\section{Results}

\subsection{Results of the search}

A total of 437 records were identified by performing the search strategy. After removing 103 duplicates, 334 articles were initially included. 324 of these were subsequently excluded because they did not meet the inclusion criteria (not RCTs: 119; animal studies: 9; wrong intervention: 31; wrong disease: 165). Among the 10 potentially eligible reports, 8 were excluded for further assessment. The 8 excluded studies by checking the full text information and telephone interviews with the original authors revealed that they were not RCTs, the details of further study could not be obtained and this trial was awaiting assessment. Therefore, this paper included two studies (included 74 Chinese participants). Flow chart for studies inclusion was listed in Figure 1.

\subsection{The included studies}

The analysis included two studies conducted in China that enrolled a total of 74 adult patients ( $\mathrm{n}=32$ and 42 , respectively) with RPGN (Deng et al., 2004; Wang et al., 2008). The ratio of male-to-female participants was 36 to 38 and the age range was from 25 to 58 years old. In the study of Deng et al. (2004), the disease duration was 2-13 $(0.87 \pm 0.62)$ months in the treatment group and 2-11 $(0.82 \pm 0.60)$ months in the control group. The disease duration in the study of Wang et al. (2008) was 3-15 (0.88 \pm 0.65$)$ months in the treatment group and 2-14 (0.84 \pm 0.62$)$ months in the control group. The diagnostic criteria for RPGN included severe hematuria, persistent oliguria, a rapid decline in kidney function, the presence of extensive glomerular crescents (usually affecting > $50 \%$ of the glomeruli) as the main histological finding (as defined by the authors), and biopsy-proven RPGN (types I to III).

In addition, we analysed the intervention in the two studies. Chinese medicinal herbs plus supportive therapies combined with specific therapies were given via oral or intravenous drip. The therapy duration was 3 months and follow-up duration was not reported in two included studies. Two studies compared Chinese medicinal herbs formulae plus supportive therapies combined with specific therapies with supportive therapies combined with specific therapies.

Moreover, we analysed the outcomes of the two stydies. Regarding primary outcomes, both studies reported the incidence of ESKD and oliguria and anuria remission. However, neither reported mortality rates. Regarding secondary outcomes, the levels of SCr, BUN, Hb, HCT and the use of immunosuppressive agents were reported in both studies. However, neither study 

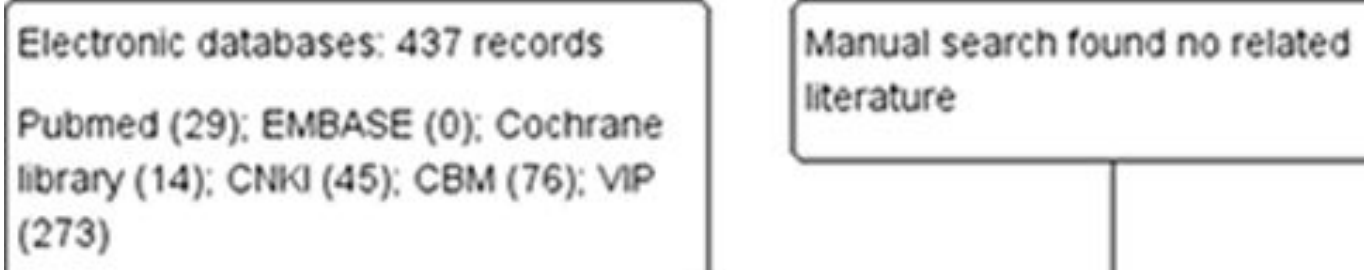
Pubmed (29); EMBASE (0); Cochrane (273)

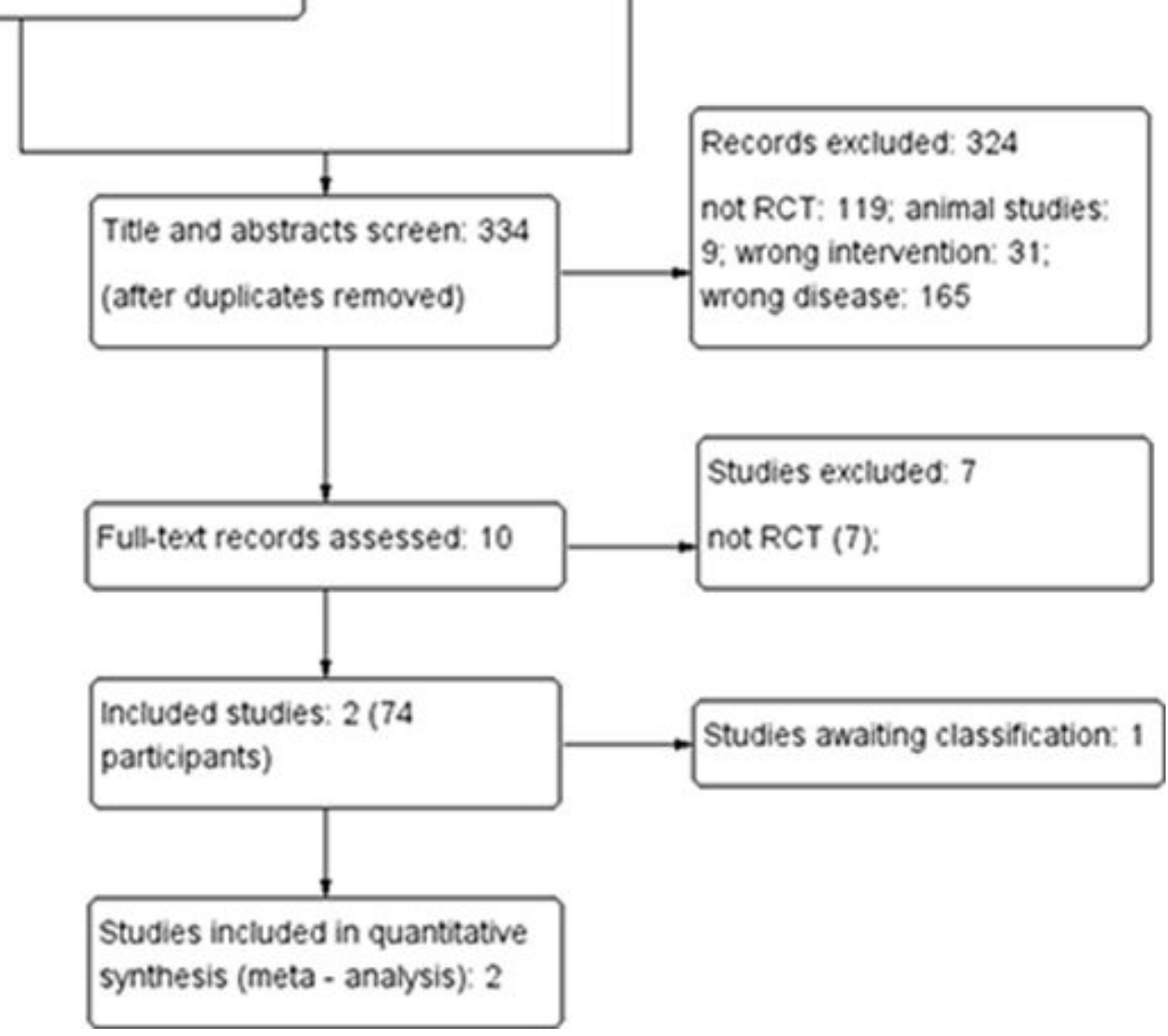

Figure 1. Summary of the search results in a flow diagram.

reported AST or ALT levels, time for 24-h urine volume to return to normal, quality of life, tongue coating and pulse status, economic index, or rate of withdrawals from the study. The characteristics of the studies were shown in detail in Table 2.

\subsection{Risk of bias in included studies}

The studies included in our review were of moderate quality. Their methodological quality with respect to the randomization sequence, blinding (of both participants and researchers, and regarding the outcomes), allocation concealment, selective outcome reporting, incomplete outcome data, and other biases (Figures 2 and 3).

\section{Allocation}

Participants were randomized using a random number table in both studies. Although the method of allocation concealment was not reported in either study, we ascertained via a telephone interview with the authors that the randomization method used in the studies. Wang et al. precluded the investigators and participants from cognizance of the group assignments of the eligible participants enrolled in the study. However, the allocation concealment was inadequate in the study of Deng et al. (2004).

\section{Ongoing studies}

We have not identified any ongoing studies in the Chinese Clinical Trial Register which would meet our pre-defined inclusion criteria.

\subsection{Effects of interventions}

The Chinese medicinal herb treatments included a self-developed clear heat and activate blood prescription and modified Chinese 
Table 2. Characteristics of included studies.

\begin{tabular}{|c|c|c|}
\hline Study & Parts & Contents \\
\hline $\begin{array}{c}\text { Deng et al. (2004) } \\
{[18]}\end{array}$ & Methods & $\begin{array}{l}\text { Randomized controlled trial (RCT): randomization mentioned, described in detail. Allocation concealment: not mentioned. } \\
\text { Follow-up: not mentioned. Study duration: } 3 \text { months. Parallel/crossover/factorial RCT: parallel RCT. Randomization } \\
\text { method: random number table. Blinding: no detailed information on blinding was offered. ITT: Not mentioned. }\end{array}$ \\
\hline
\end{tabular}
Participants Setting: outpatients. Country: China. Number (randomized/analyzed): treatment group (16/16); control group (16/16). Treatment group: 16 (6 males; 37.5\%; 10 females; $62.5 \%$ ), age $28-57$ (average $36.1 \pm 18.3$ ) years old; disease duration: 2 - 13 (average $0.87 \pm 0.62$ ) months. Control group: 16 (7 males; $43.8 \%$; 9 females; $56.2 \%$ ), age $31-76$ (average $37.4 \pm$ 17.5) years old; disease duration: $2-13$ (average $0.87 \pm 0.62$ ) months. Biopsy-proven membranous nephropathy (types I, II and III).

Treatment group: Self-Developed clear hear and activate blood prescription plus supportive therapies combined with specific therapies. Self-Developed clear hear and activate blood prescription: Baihuasheshecao 30 g, Rendongteng $30 \mathrm{~g}$, Zihuadiding 30 g, Chishao 15 g, Shengdi 15 g, Huangjing 15 g, Dangshen 30 g, Danshen 30 g, Zhidahuang 12 g, and Huoxiang $12 \mathrm{~g}$ boiled in $3 \mathrm{~L}$ of water and decocted to $300 \mathrm{~mL}$. Orally twice daily (bid)/ one dose daily for 3 months. Supportive therapies: Consistent with the control group. Specific therapies: 15 cases were given methylprednisolone shock treatment (dose of $160-480 \mathrm{mg} / \mathrm{d}$ ) for 3 days, with the dose gradually reduced afterward; they were then switched to oral prednisone $0.8-1.0 \mathrm{mg} / \mathrm{kg} / \mathrm{d}$ after $7-14$ days. The prednisone dose was then reduced according to the conventional reduction method. While on the reduced Interventions prednisone dose, four cases also underwent cyclophosphamide shock treatment $1.5 \mathrm{~g} / \mathrm{d}$ oral, and eight also underwent cyclophosphamide shock treatment $0.8 \mathrm{~g} /$ month. In the treatment process, only one case did not use immunosuppressant therapy. Control group: supportive therapies combined with specific therapies. Supportive therapies including blood pressure control using amlodipine besylate tablets $5-10 \mathrm{mg} / \mathrm{d}$, subcutaneous erythropoietin $4000-6000 \mathrm{U} /$ week when $\mathrm{Hb} \leq$ $90 \mathrm{~g} / \mathrm{L}$, and hemodialysis when SCr $>707 \mu \mathrm{m}$. Specific therapies: 16 cases were given methylprednisolone shock treatment (dose of 320-1000 mg/d) for 3 days, with the dose gradually reduced afterward; they were then switched to oral prednisone $0.8-1.0 \mathrm{mg} / \mathrm{kg} / \mathrm{d}$ after $7-14$ days. The prednisone dose was then reduced according to the conventional reduction method. While on the reduced prednisone dose, three patients were also given mycophenolate mofetil $1.5-2.0 \mathrm{~g} / \mathrm{d}$ oral, and 11 cases also underwent cyclophosphamide shock treatment $0.8 \mathrm{~g} / \mathrm{month}$. In the treatment process, 16 patients used immunosuppressant therapy.

Outcomes $\quad$ 1. ESKD; 2. SCr, BUN; 3. Hb, HCT; 4. Utilization number of immunosuppressive agents (including Methylprednisolone and prednisone, Mycophenolate Mofetil, and Cyclophosphamide); 5. Oliguria or anuria remission.

1. Mortality: not mentioned; 2. ALT, AST: not mentioned; 3.24 hour urine volume return to normal time: not mentioned; 4. Quality of life: not mentioned; 5. Tongue coat and pulse condition: not mentioned; 6. Economic index: not mentioned; 7. 4. Quality of life: not mentioned; 5 . Tongue coat and pulse condition: not mentioned; 6 . Economic index: not mentioned; 7.
Adverse events: not mentioned; 8. Withdrawal: not mentioned; 9. Project support: not mentioned; 10. Overall, participant demographic data were similar between groups; 11 . The protocol: not mentioned; 12 . Informed consent: not mentioned, but we confirmed by telephone that an informed consent form was obtained from each participant; 13. Ethics committee approval: not mentioned.

Wang et al., 2008 [19]

Methods

Participants

Interventions

Outcomes

Notes
RCT: randomization mentioned, described in detail. Allocation concealment: not mentioned, but a telephone interview with the author revealed that randomization method described could not allow investigators/participants to know or influence intervention group before eligible participant entered in the study. Follow-up: not mentioned. Study duration: 3 months. Parallel/crossover/factorial RCT: parallel RCT. Randomization method: random number table. Blinding: no detailed information on blinding was offered. A telephone interview with the author revealed that participants did not know drugs used status and blinding of outcome assessment was not used. ITT: Not mentioned

Setting: outpatients. Country: China. Number (randomized/analyzed): treatment group (21/21); control group (21/21). Treatment group: 21 (12 males; 57.1\%; 9 females; $42.9 \%$ ), age 25 - 57 (average $34.6 \pm 17.3$ ) years old; disease duration: $3-15$ (average $0.88 \pm 0.65$ ) months. Control group: 21 ( 11 males; $52.4 \% ; 10$ females; $47.6 \%$ ), age $27-56$ (average $35.4 \pm$ 18.2) years old; disease duration: 2 - 14 (average $0.84 \pm 0.62$ ) months. Biopsy proven membranous nephropathy (types I, II and III).

Treatment group: Chinese medicinal herbs formulae plus supportive therapies combined with specific therapies. 1.Pattern of accumulation of dampness-heat and blood stasis in the kidney collateral: Baihuasheshecao 20 g, Pugongying 12 g, Rendongteng 12 g, Zihuadiding 15 g, Danshen 15 g, Chishao 12 g, Taoren 12 g, Shengdi 18 g, Mugua 12 g, Zhidahuang $6 \mathrm{~g}$, Gancao $6 \mathrm{~g} ; 2$. Pattern of kidney yin deficiency and blood stasis in the kidney collateral: Shengdi 18 g, Maidong 12 g, Shanzhuyu 10 g, Huangjing 15 g, Shihu 15 g, Guiban 20 g, Zhimu 12 g, Duzhong 15 g, Huangbai $12 \mathrm{~g}$, Danshen $15 \mathrm{~g}$, Chishao $12 \mathrm{~g}$, Zhidahuang $6 \mathrm{~g}$; 3. Pattern of dual deficiency of the spleen-kidney and blood stasis in the kidney collateral: Huangqi 20 g, Huaishanyao 18 g, Chuanxiong 10 g, Gegen 15 g, Huangjing 15 g, Shanzhuyu 12 g, Xianlingpi 12 g, Bajitian 12 g, Taoren 12 g, Honghua 6 g, Baizhu 15 g, Zhidahuang 6 g. Supportive therapies: Consistent with the control group. Specific therapies: 19 cases were given methylprednisolone shock treatment (dose of $160-480 \mathrm{mg} / \mathrm{d}$ ) for 3 days, with the dose gradually reduced afterward; they were then switched to oral prednisone $0.8-1.0 \mathrm{mg} / \mathrm{kg} / \mathrm{d}$ after $7-14$ days. The prednisone dose was then reduced according to the conventional reduction method. While on the reduced prednisone dose, five cases were also given mycophenolate mofetil $1.5 \mathrm{~g} / \mathrm{d}$ oral, and nine cases also underwent cyclophosphamide shock treatment $0.8 \mathrm{~g} / \mathrm{month}$. In the treatment process, 2 causes did not use immunosuppressant therapy. Control group: supportive therapies combined with specific therapies. Supportive therapies including blood pressure control by using amlodipine besylate tablet $5-10 \mathrm{mg} / \mathrm{d}$, subcutaneous erythropoietin 4000-6000U/week when $\mathrm{Hb} \leq 90 \mathrm{~g} / \mathrm{L}$, and hemodialysis when SCr $>707 \mu \mathrm{m}$. Specific therapies: 21 cases were given methylprednisolone shock treatment (dose of $320-1000 \mathrm{mg} / \mathrm{d}$ ) for 3 days, with the dose gradually reduced afterward; they were then switched to oral prednisone $0.8-1.0 \mathrm{mg} / \mathrm{kg} / \mathrm{d}$ after $7-14$ days. The prednisone dose was then reduced according to the conventional reduction method. While on the reduced prednisone dose, three cases were also given mycophenolate mofetil $1.5-2.0 \mathrm{~g} / \mathrm{d}$ oral, 11 cases also underwent cyclophosphamide shock treatment $0.8 \mathrm{~g} / \mathrm{month}$. In the

treatment process, 21 cases used immunosuppressant therapy.
1. ESKD; 2. SCr, BUN; 3. Hb, HCT; 4. Utilization number of immunosuppressive agents (including Methylprednisolone and prednisone, Mycophenolate Mofetil, and Cyclophosphamide); 5. Oliguria or anuria remission.

1. Mortality: not mentioned; 2. ALT, AST: not mentioned; 3.24 hour urine volume return to normal time: not mentioned; 4. Quality of life: not mentioned; 5. Tongue coat and pulse condition: not mentioned; 6 . Economic index: not mentioned; 7. Adverse events: not mentioned; 8. Withdrawal: not mentioned; 9. Project support: not mentioned; 10. Overall, participant demographic data were similar between groups; 11 . The protocol: not mentioned; 12 . Informed consent: not mentioned, but an informed consent form was obtained from each participant by telephone confirmed; 13 . Ethics committee approval: not mentioned.

\footnotetext{
Notes: RCTs, randomized controlled trials; ITT, intention-to-treat; ESKD, end-stage kidney disease; SCr, serum creatinine; Hb, haemoglobin; HCT, Hematocrit; PRGN, Rapidly progressive glomerulonephritis; BUN, urea nitrogen; AST, aspartate transaminase; ALT, alanine transaminase.
} 


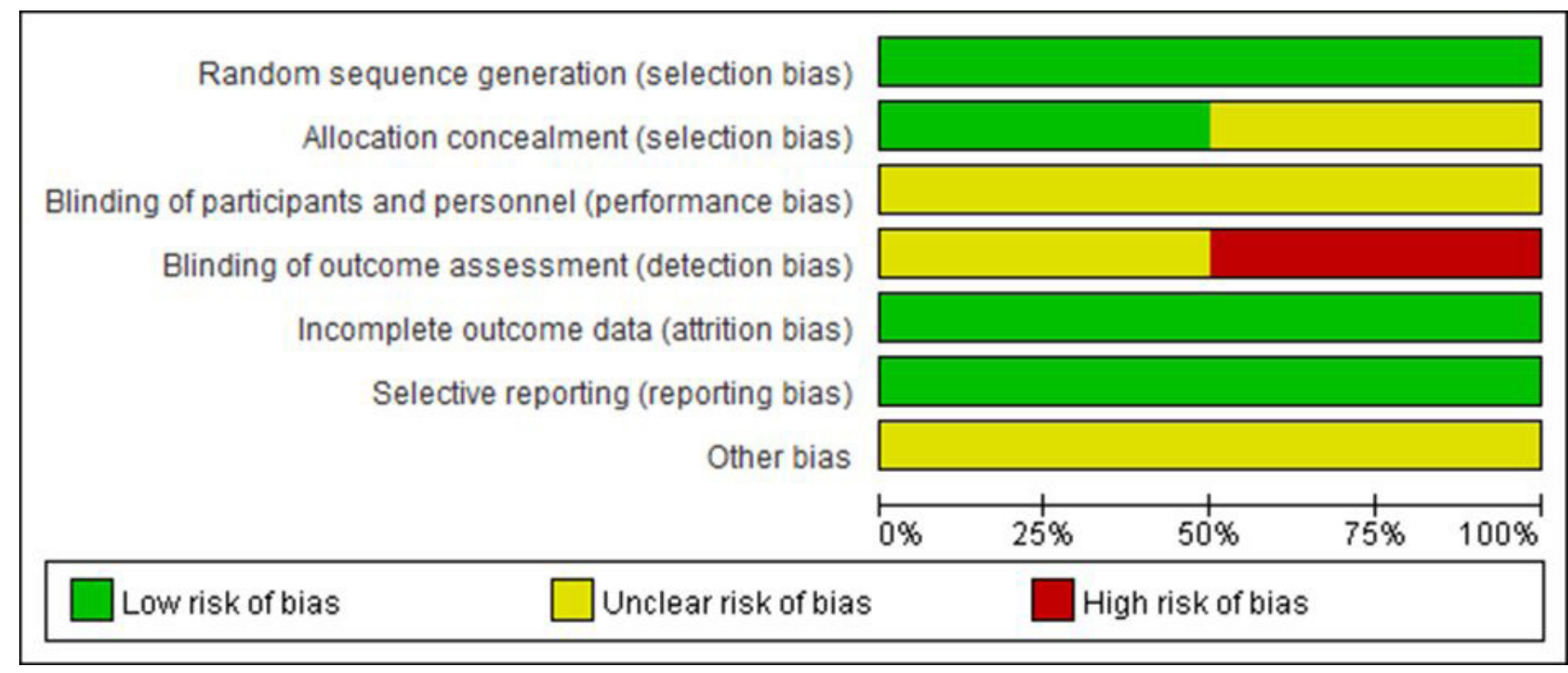

Figure 2. Risk of bias graph: review authors' judgements about each risk of bias item presented as percentages across all included studies.

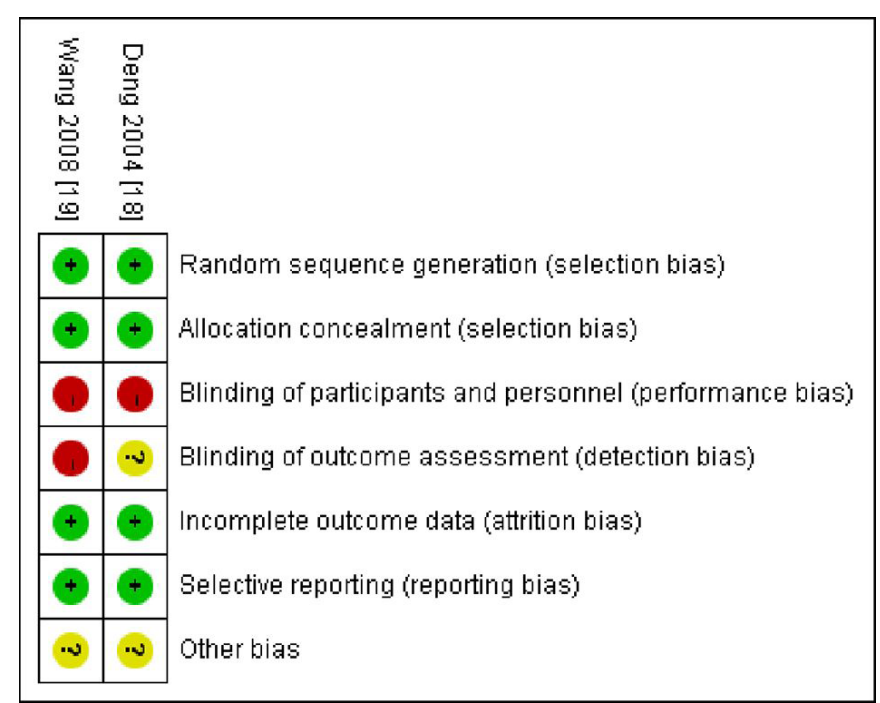

Figure 3. Risk of bias summary: review authors' judgements about each risk of bias item for each included study.

medicinal herb prescriptions based on the syndrome differentiation, including accumulated dampness-heat and blood stasis, kidney yin deficiency and blood stasis, and deficiency in the spleen-kidney and blood stasis in the kidney. Supportive therapies included blood pressure control using amlodipine besylate, subcutaneous injection of erythropoietin, and hemodialysis. Specific therapies included methylprednisolone, prednisone, mycophenolate mofetil, and cyclophosphamide. The two studies investigated Chinese medicinal herbs plus supportive therapies combined with specific therapies versus supportive therapies combined with specific therapies.

\section{Primary outcomes}

There was no significant difference in ESKD prevention between between Chinese medicinal herbs plus supportive therapies combined with specific therapies and supportive therapies combined with specific therapies. (Figure 4; Analysis 1.1; RR 0.50 ; $95 \% \mathrm{CI}, 0.17$ to $\left.1.51 ; \mathrm{I}^{2}=0 \%\right)$. In addition, there was also no significant difference in oliguria or anuria remission between the two therapies (Figure 5; Analysis 1.2; RR, 1.97; 95\% CI, 0.89 to $\left.4.37 ; \mathrm{I}^{2}=10 \%\right)$.

\section{Secondary outcomes}

Chinese medicinal herbs plus supportive therapies combined with specific therapies significantly decreased $\mathrm{SCr}$ when compared to supportive therapies combined with specific therapies alone (Figure 6; Analysis 1.3; MD, -145.93 $\mu \mathrm{mol} / \mathrm{L}$; $95 \% \mathrm{CI},-236.75$ to $\left.-55.11 ; \mathrm{I}^{2}=0 \%\right)$. Besides, Chinese medicinal herbs plus supportive therapies combined with specific therapies did not significantly decrease BUN when compared to supportive therapies combined with specific therapies alone (Figure 7; Analysis 1.4; MD, $-4.45 \mathrm{mmol} / \mathrm{L}$; 95\% CI, -9.00 to $\left.0.11 ; \mathrm{I}^{2}=0 \%\right)$. However, there was no significant difference in $\mathrm{Hb}$ between the two therapies (Figure 8; Analysis 1.5; MD, $0.19 \mathrm{~g} / \mathrm{L} ; 95 \% \mathrm{CI},-20.87$ to $21.25 ; \mathrm{I}^{2}=0 \%$ ). Similarly, there was no significant difference in HCT between the two therapies (Figure 9; Analysis 1.6; MD, $-0.39 \%, 95 \% \mathrm{CI},-5.91$ to 5.13 ; $\mathrm{I}^{2}$ $=0 \%)$. Inaddition, Chinese medicinal herbs plus supportive therapies combined with specific therapies significantly decreased utilization number of methylprednisolone and prednisone when compared to supportive therapies combined with specific therapies alone (Figure 10; Analysis 1.7; MD, -1.64g; 95\% CI, -1.87 to $\left.-1.40 ; \mathrm{I}^{2}=0 \%\right)$. Moreover, Chinese medicinal herbs plus supportive therapies combined with specific therapies did not significantly decrease utilization number of cyclophosphamide when compared to supportive therapies combined with specific therapies alone (Figure 11; Analysis, 1.8; MD, $-0.87 \mathrm{~g}$; 95\% CI, -1.95 to $0.21 ; \mathrm{I}^{2}=74 \%$ ). Furthermore, Chinese medicinal herbs plus supportive therapies combined with specific therapies did not significantly decrease utilization number of mycophenolate 


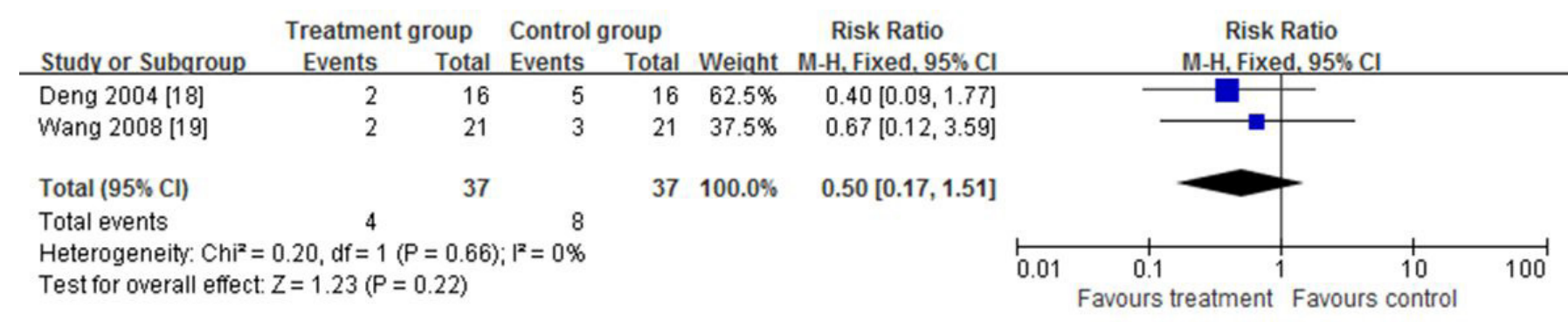

Figure 4. Comparison between the two therapies: outcome 1 ESKD (analysis 1.1).

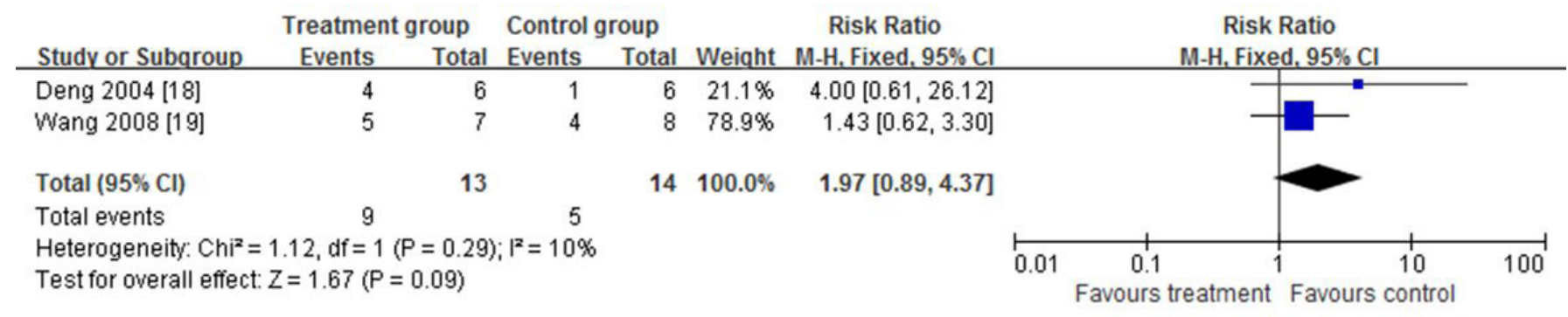

Figure 5. Comparison between the two therapies: outcome 2 oliguria or anuria remission (analysis 1.2).

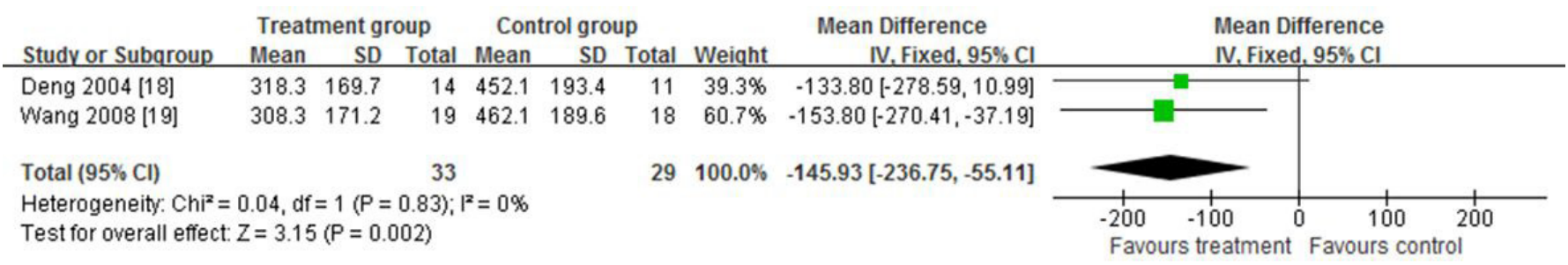

Figure 6. Comparison between the two therapies: outcome $3 \mathrm{SCr}$ (analysis 1.3).

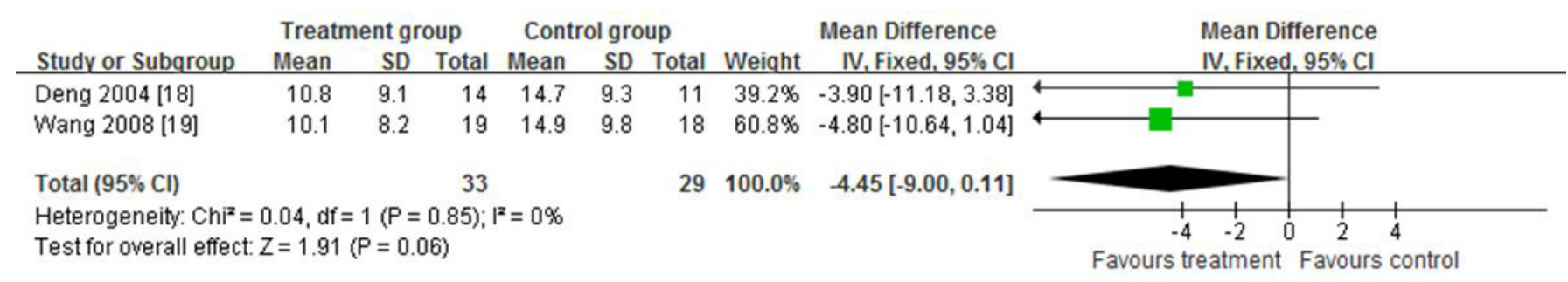

Figure 7. between the two therapies: outcome 4 BUN (analysis 1.4).

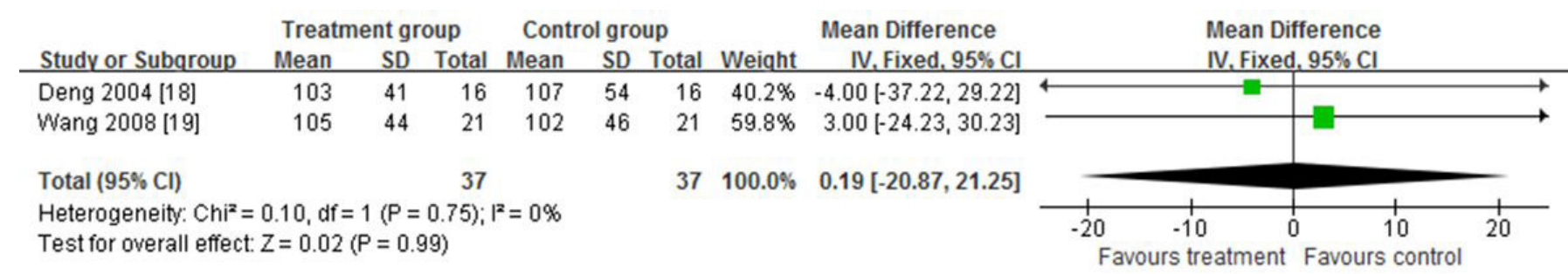

Figure 8. Comparison between the two therapies: outcome $5 \mathrm{Hb}$ (analysis 1.5).

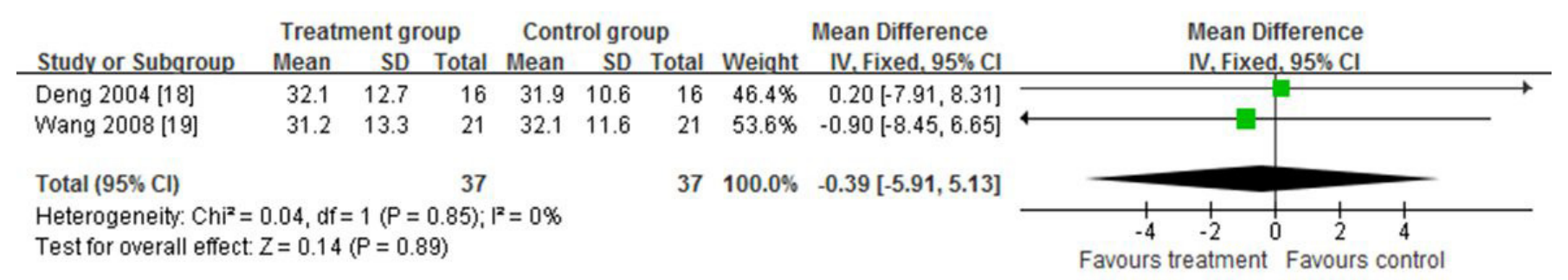

Figure 9. Comparison between the two therapies: outcome 6 HCT (analysis 1.6). 
mofetil when compared to supportive therapies combined with specific therapies alone (Figure 12; Analysis 1.9; MD, 5.63g; $95 \% \mathrm{CI},-19.86$ to $\left.31.12 ; \mathrm{I}^{2}=0 \%\right)$.

\section{Other outcomes}

In two studies, neither therapies did report all-cause mortality, levels of AST and ALT, time for the 24-hour urine volume to return to normal, quality of life, tongue coat and pulse condition, economic index, adverse events, and withdrawal. The "GRADE profiler" of the Cochrane Collaboration Network was used to classify the systematic review results. The quality of evidence was low (Table 3).

\section{Discussion}

Our analysis included two RCTs conducted in China which enrolled a total of 74 Chinese participants. We found some evidences of efficacy of Chinese medicinal herbs used in combination with supportive and specific therapies for RPGN through a reduction in SCr levels and the methylprednisolone and prednisone doses required. However, the evidences were not sufficient to confirm a positive effect of the combination treatment on levels of $\mathrm{Hb}$ and $\mathrm{HCT}$, oliguria or anuria status, BUN levels, incidence of ESRD, or cyclophosphamide and mycophenolate use between the two therapies. Given the small sample sizes of the included studies, we were not able to assess the relationship between RPGN type and TCM efficacy, and the overall evidences supporting the safety and efficacy of Chinese medicinal herbs in adult patients with RPGN were limited.

The quality of the evidence and reportage of the findings were suboptimal in both studies (Table 3). Thus, the results were not robust, although further analysis could improve our confidence in the findings. The studies did not have sufficient power, and the methodologies were either well-described or flawed. For example, neither study mentioned allocation concealment, although a telephone interview with the authors revealed that in the study of Wang et al., group allocation was blinded. Moreover, the blinding procedures were not discussed in either study. Based on the telephone interview, we were able to know that Wang et al. used a double-blind procedure (i.e., blinding of both participants and investigators). However, outcomes were not blinded. Consequently, the risk of detection bias was high. Regarding the study of Deng et al. (2004), we were unable to obtain sufficient additional information for a comprehensive assessment.

In conclusion, Chinese medicinal herbs may reduce $\mathrm{SCr}$ levels and the utilization number of methylprednisolone and prednisone in adult patients with RPGN, suggesting that these herbs were somewhat effective in the treatment of RPGN in

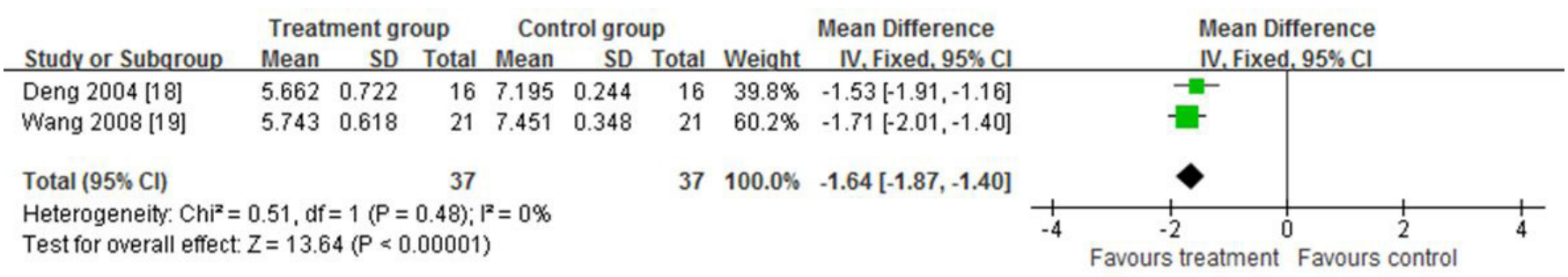

Figure 10. Comparison between the two therapies: outcome 7 utilization number of methylprednisolone and prednisone (analysis 1.7).

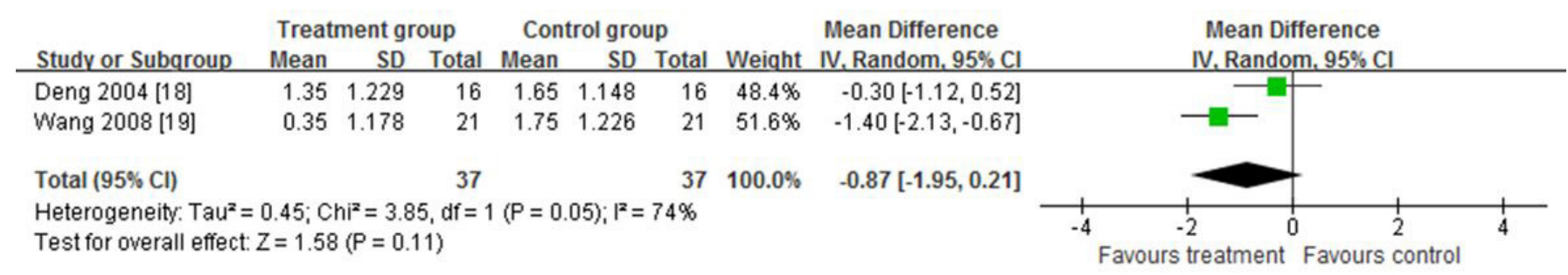

Figure 11. Comparison between the two therapies: outcome 7 utilization number of cyclophosphamide (analysis 1.8).

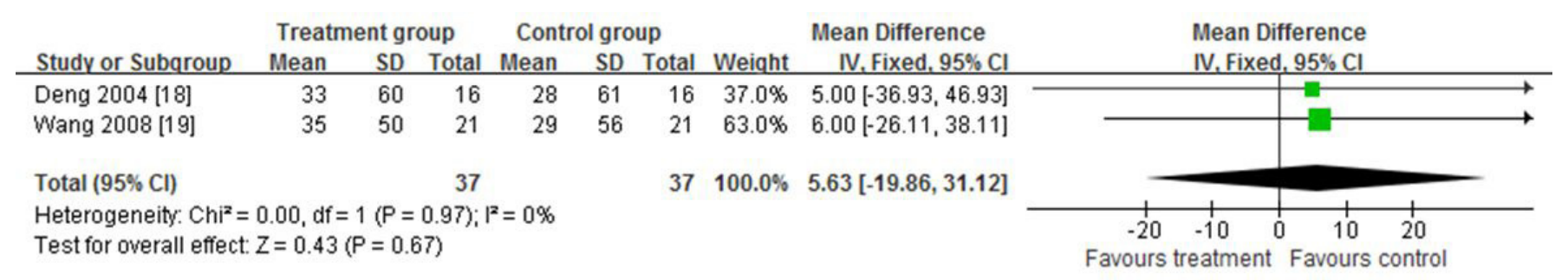

Figure 12. Comparison between the two therapies: outcome 7 utilization number of mycophenolate mofetil (analysis 1.9). 
Table 3. GRADE Quality of Evidence.

Chinese medicinal herbs plus supportive therapies combined with specific therapies versus supportive therapies combined with specific therapies for rapidly progressive glomerulonephritis in adults

Patient or population: patients with rapidly progressive glomerulonephritis in adults

Settings: inpatients and outpatients

Intervention: Chinese medicinal herbs plus supportive therapies combined with specific therapies versus supportive therapies combined with specific therapies

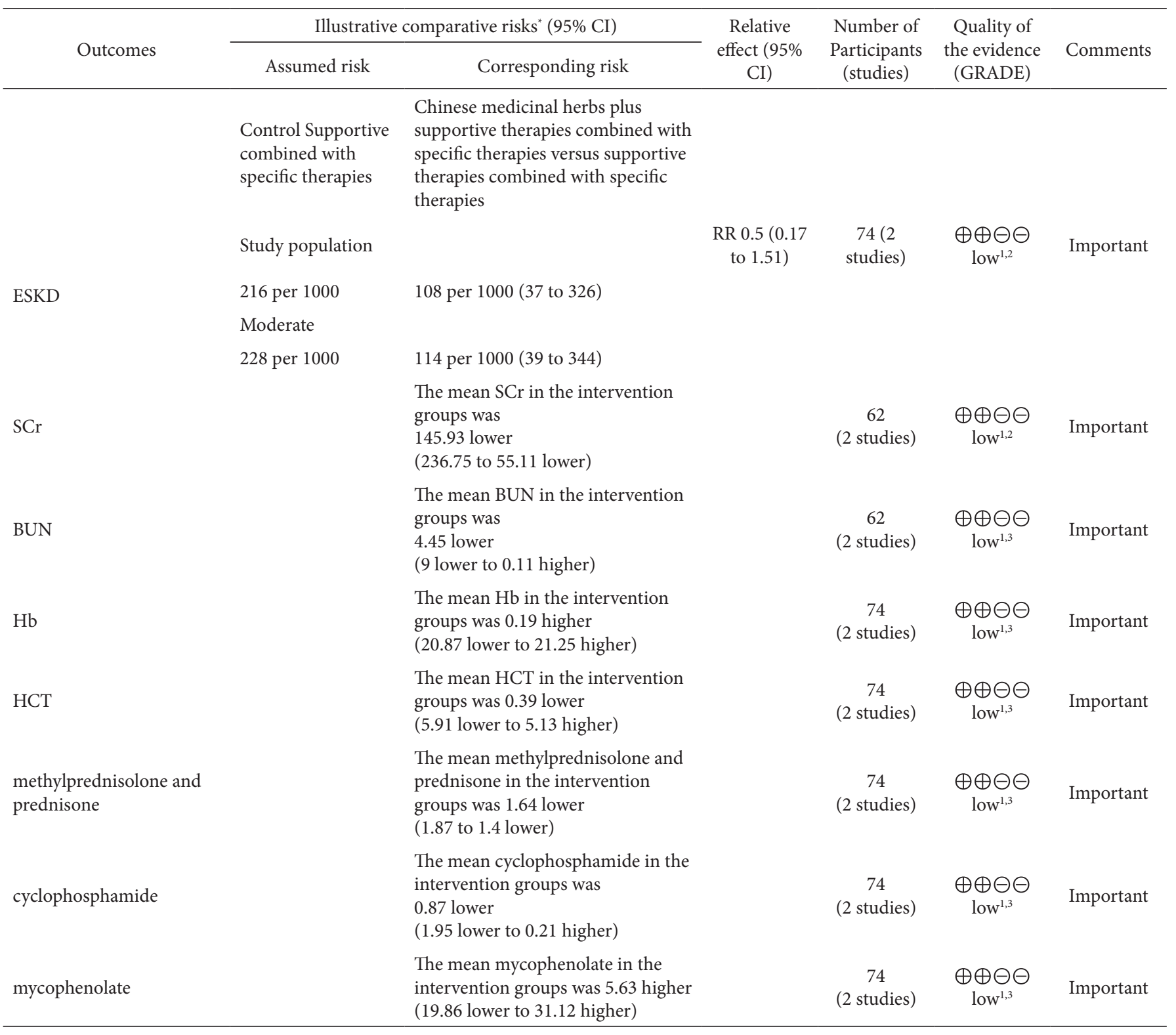

* The basis for the assumed risk (e.g. the median control group risk across studies) was provided in footnotes. The corresponding risk (and its $95 \% \mathrm{CI}$ ) was based on the assumed risk in the comparison group and the relative effect of the intervention (and its 95\% CI). CI: Confidence interval. RR: Risk ratio. GRADE Working Group grades of evidence: high quality, further research was very unlikely to change our confidence in the estimate of effect; moderate quality, further research was likely to have an important impact on our confidence in the estimate of effect and may change the estimate; low quality, further research was very likely to have an important impact on our confidence in the estimate of effect and was likely to change the estimate; very low quality, we were very uncertain about the estimate. ${ }^{1}$ High risk of detection bias; ${ }^{2}$ Few studies included; ${ }^{3}$ Two studies included.

this population. However, the evidence was insufficient to confirm that Chinese medicinal herbs increased the levels of $\mathrm{Hb}$ and $\mathrm{HCT}$, relieve oliguria or anuria, or reduced BUN levels, the incidence of ESRD, or cyclophosphamide and mycophenolate use. Our analysis was based on the findings of the two studies of moderate methodological quality. Larger, multicenter studies of high methodological quality are needed to further examine the usefulness of Chinese medicinal herbs 
for the treatment of RPGN in adults. Future studies should include patients with RPGN being treated with prescribed Chinese medicinal herb preparations.

\section{Ethic approval}

All procedures performed in studies involving human participants were in accordance with the ethical standards of the institutional and/or national research committee and with the 1964 Helsinki declaration and its later amendments or comparable ethical standards. This study is approved by relevant Ethics Committee.

\section{Informed Consent}

Written informed consent was obtained.

Conflict of Interests: The authors declared no conflict of interests.

\section{References}

Arimura, Y., Muso, E., Fujimoto, S., Hasegawa, M., Kaname, S., Usui, J., Ihara, T., Kobayashi, M., Itabashi, M., Kitagawa, K., Hirahashi, J., Kimura, K., \& Matsuo, S. (2016). Evidence-based clinical practice guidelines for rapidly progressive glomerulonephritis 2014. Clinical and Experimental Nephrology, 20(3), 322-341. http://dx.doi.org/10.1007/ s10157-015-1218-8. PMid:27099135.

Cattran, D.C., Feehally, J., Cook, H.T., \& Liu, Z.-H. (2012). Kidney Disease: Improving Global Outcomes (KDIGO) Glomerulonephritis Work Group. KDIGO Clinical Practice Guideline for Glomerulonephritis. Kidney International Supplements, 2(2), 139-274.

Chembo, C. L., Marshall, M. R., Williams, L. C., Walker, R. J., Lynn, K. L., Irvine, J., \& Pilmore, H. L. (2015). Long-term outcomes for primary glomerulonephritis: New Zealand Glomerulonephritis Study. Nephrology, 20(12), 899-907. http://dx.doi.org/10.1111/ nep.12538. PMid:26096749.

Chen, Y. P. (2005). Integration of traditional Chinese and Western medicine for crescent nephritis. Chinese Journal of Integrated Traditional and Western Nephrology, 6(5), 249-252.

Couser, W. G. (1988). Rapidly progressive glomerulonephritis: classification, pathogenetic mechanisms, and therapy. American Journal of Kidney Diseases, 11(6), 449-464. http://dx.doi.org/10.1016/ S0272-6386(88)80079-9. PMid:3287904.

Deng, Y. Y., Chen, Y. P., Wang, L., Hu, Z.Y., Jin, Y.M., Shen, L.M., Zhu, R., \& Zhong, Y.W. (2004). Heat-clearing and Blood-activating prescription for middle-advanced crescent nephritis clinical study. Chinese Journal of Integrated Traditional and Western Medicine, 24(12), 1084-1086.

Higgins, J. P., \& Green, S., (Eds.) (2011). Cochrane Handbook for Systematic Reviews of Interventions Version 5.1.0. [updated March 2011]. London, UK: The Cochrane Collaboration. Retrieved from www.cochrane-handbook.org

Higgins, J. P., \& Thompson, S. G. (2002). Quantifying heterogeneity in a meta-analysis. Statistics in Medicine, 21(11), 1539-1558. http:// dx.doi.org/10.1002/sim.1186. PMid:12111919.

Jennette, J. C. (2003). Rapidly progressive crescentic glomerulonephritis. Kidney International, 63(3), 1164-1177. http://dx.doi.org/10.1046/ j.1523-1755.2003.00843.x. PMid:12631105.

Ka, S. M., Kuo, Y. C., Ho, P. J., Tsai, P. Y., Hsu, Y. J., Tsai, W. J., Lin, Y. L., Shen, C. C., \& Chen, A. (2010). (S)-Armepavine from
Chinese medicine improves experimental autoimmune crescentic glomerulonephritis. Rheumatology, 49(10), 1840-1851. http://dx.doi. org/10.1093/rheumatology/keq164. PMid:20551296.

Kannan, S., \& Mattoo, T. K. (2001). Diffuse crescentic glomerulonephritis in bacterial endocarditis. Pediatric Nephrology, 16(5), 423-428. http:// dx.doi.org/10.1007/s004670000550. PMid:11405117.

Liu, C. P., Kuo, Y. C., Shen, C. C., Wu, M. H., Liao, J. F., Lin, Y. L., Chen, C. F., \& Tsai, W. J. (2007). (S)-armepavine inhibits human peripheral blood mononuclear cell activation by regulating Itk and PLCgamma activation in a PI-3K-dependent manner. Journal of Leukocyte Biology, 81(5), 1276-1286. http://dx.doi.org/10.1189/ jlb.0106056. PMid:17284681.

Liu, C. P., Tsai, W. J., Shen, C. C., Lin, Y. L., Liao, J. F., Chen, C. F., \& Kuo, Y. C. (2006). Inhibition of (S)-armepavine from Nelumbo nucifera on autoimmune disease of MRL/MpJ-lpr/lpr mice. European Journal of Pharmacology, 531(1-3), 270-279. http://dx.doi.org/10.1016/j. ejphar.2005.11.062. PMid:16413531.

Liu, G., Ma, X. Z., Zou, W. Z., Wang, M., \& Wang, H. Y. (2004). The composition of kidney biopsy results in ten years. Journal of Clinical Internal Medicine, 12:834-838.

Lu, X. (2018). Research progress of Chinese and Western medicine in the treatment of chronic glomerulonephritis. World latest medical information abstracts, (57), 64-65-67.

Moroni, G., \& Ponticelli, C. (2014). Rapidly progressive crescentic glomerulonephritis: early treatment is a must. Autoimmunity Reviews, 13(7), 723-729. http://dx.doi.org/10.1016/j.autrev.2014.02.007. PMid:24657897.

Nachman, P. H., Jennette, J. C., \& Falk, R. J. (2011). Primary Glomerular disease. In M. W. Taal, G. M. Chertow, P. A. Marsden, K. Skorecki, A. S. L. Yu, B. M. Brenner (Eds.), Brenner \& Rector's the Kidney (9th ed., pp. 1100-91). Philadelphia: Saunders. http://dx.doi.org/10.1016/ B978-1-4160-6193-9.10031-4.

Okpechi, I. G., Ameh, O. I., Bello, A. K., Ronco, P., Swanepoel, C. R., \& Kengne, A. P. (2016). Epidemiology of histologically proven Glomerulonephritis in Africa: A systematic review and metaanalysis. PLoS One, 11(3), e0152203. http://dx.doi.org/10.1371/ journal.pone.0152203. PMid:27011216.

Parmar, M. S., \& Bhimji, S. S. (2018) StatPearls. Treasure Island (FL): StatPearls Publishing.

Perkowska-Ptasinska, A., Bartczak, A., Wagrowska-Danilewicz, M., Halon, A., Okon, K., Wozniak, A., Danilewicz, M., Karkoszka, H., Marszalek, A., Kowalewska, J., Mroz, A., Korolczuk, A., Oko, A., Debska-Slizien, A., Naumnik, B., Hruby, Z., Klinger, M., Ciechanowski, K., Myslak, M., Sulowicz, W., Rydzewski, A., Wiecek, A., Manitius, J., Gregorczyk, T., Niemczyk, S., Nowicki, M., Gellert, R., Stompor, T., Wieliczko, M., Marczewski, K., Paczek, L., Rostkowska, O., Deborska-Materkowska, D., Bogdanowicz, G., Milkowski, A., \& Durlik, M., and the Polish Society of Nephrology (2017). Clinicopathologic correlations of renal pathology in the adult population of Poland. Nephrology, Dialysis, Transplantation, 32(Suppl. 2upl. Suppl. 2), ii209-ii218. PMid:28339709.

Quiroga, B., Vega, A., Rivera, F., \& Lopez-Gomez, J. M. (2015). Crescentic glomerulonephritis: data from the Spanish Glomerulonephritis Registry. Internal Medicine Journal, 45(5), 557-562. http://dx.doi. org/10.1111/imj.12725. PMid:25684569.

Schena, F. P., and the The Italian Group of Renal Immunopathology (1997). Survey of the Italian Registry of Renal Biopsies. Frequency of the renal diseases for 7 consecutive years. The Italian Group of Renal Immunopathology. Nephrology, Dialysis, Transplantation, 12(3), 418-426. http://dx.doi.org/10.1093/ndt/12.3.418. PMid:9075118.

Succar, L., Boadle, R. A., Harris, D. C., \& Rangan, G. K. (2016). Formation of tight junctions between neighboring podocytes is an early 
ultrastructural feature in experimental crescentic glomerulonephritis. International Journal of Nephrology and Renovascular Disease, 24(9), 297-312. http://dx.doi.org/10.2147/IJNRD.S113071. PMid:27920570.

van Daalen, E. E., Jennette, J. C., McAdoo, S. P., Pusey, C. D., Alba, M. A., Poulton, C. J., Wolterbeek, R., Nguyen, T. Q., Goldschmeding, R., Alchi, B., Griffiths, M., de Zoysa, J. R., Vincent, B., Bruijn, J. A., \& Bajema, I. M. (2018). Predicting Outcome in Patients with Anti-GBM Glomerulonephritis. Clinical Journal of Americian Society of Nephrology, 13(1), 63-72. http:// dx.doi.org/10.2215/CJN.04290417. PMid:29162595.

Wang, H., Zhang, W. P., \& Liao, H. (2008). Integration of traditional Chinese and Western medicine for 21 cases middle-advanced crescent nephritis clinical study. Journal of Sichuan of Traditional Chinese Medicine, 26(4), 57-58.
Wang, W. W. (2003). Experience of Professor Chen Yi-Ping on the treatment of crescentic nephritis by Integrated Chinese and Western Medicine. Chinese Journal of Integrated Traditional and Western Nephrology, 4(10), 563-564.

Wang, W. W., \& Chen, Y. P. (2004). Treatment of crescentic Nephritis by integrated chinese and western medicine. Shanghai Journal of Traditional Chinese Medicine, 38(4), 20-21.

Xu, Y. (1999). Experience of Professor Liu Bao-hou on the treatment of rapidly progressive crescentic nephritis. Gansu Journal of Traditional Chinese Medicine, 12(6), 11-13.

Zhong, Y. (2002). Integration of traditional Chinese and Western medicine for 21 cases rapidly progressive glomerulonephritis clinical observation. Hunan Guiding of Traditional Chinese Medicine and Pharmacology, 8(4), 179. 